\title{
Understanding Mucormycosis
}

\author{
Sumit Sharma* \\ Department of ENT, Mayo Institute of Medical Sciences, India
}

Submission: October 30, 2017; Published: November 10, 2017

*Corresponding author: Sumit Sharma, Department of ENT, Royale Medical Centre, L 3/67, Sector-D, Near Happy World Girls College, Aliganj, Lucknow, 226024, Tel: 9839178240; Email: entsumit@rediffmail.com

\section{Introduction}

Mucormycosis is a life-threatening infection that occurs in patients who are immunocompromised like uncontrolled diabetes mellitus, in particular when presenting with ketoacidosis (DKA), and other forms of acidosis, haematological malignancies, Bone marrow and solid organ transplantation, Deferoxamine therapy, Corticosteroid therapy, or other underlying conditions impairing the immune system [1]. As the incidence of risk factors are increasing the number of patients at risk for this deadly infection is increasing and despite aggressive therapy mortality rates are very high. Most of the cases of mucormycosis has been reported in the past two decades, and has become the third most common invasive fungal infection in patients with hematological malignancies and organ transplantations [1]. Although uncommon but the infection is also seen in immune-competent subjects especially after trauma (e.g., soldiers in combat operations and patients with injuries due to natural disasters) [1]. In most of the series the infection in immunecompetent subjects is commonly reported in Skin followed by Rhino-cerebral region.

The Mucorales is the largest and best studied order of zygomycete fungi. The Mucorales, which is the core group of the traditional Zygomycota, have been recently reclassified into the subphylum Mucoromycotina of the Glomeromycota phylum of the kingdom Fungi. This new classification does not include Zygomycota, because the authors consider the phylum polyphyletic, indeed the name zygomycosis, which encompassed infections caused by members of Mucorales and Entomophthorales, has become obsolete [2] although some are still using the older classification. Mucoralean fungi are ubiquitous, predominantly saprobic soil organisms on decaying organic material but parasites of plants, fungi and animals also are known [3].

Mucoralean fungi are typically fast-growing, and their wide hyphae lack septa (multiperforate septa are present only in sporangiophores and gametangia). The hyphae grow mostly within the substrate. Sporangiophores are upright (simple or ramified) hyphae that support sac-like sporangia filled with asexual sporangiospores [4]. The hyphae are wider than Aspergillus species and branch irregularly. The angle of branching is greater than other organisms, and usually approaches $90^{\circ}$ [5]. Their sexual reproduction results the formation of zygospores, whereas asexual reproduction results with nonmotile sporangiospores. They are ubiquitous in nature, being found in food, vegetation, and soil. The majority of the invasive diseases are caused by genera of the Mucoraceae family, and the resulted disease is called mucormycosis [2].

Different species of mucorales exhibit different virulism, this may provide insight as to why some species show aggressive invasive disease while other species show infrequent mortality, despite environmental ubiquity. Cunninghamella bertholettiae carries the highest mortality rates among reported cases of infection with Mucorales. However, little is known about the properties of this organism that contribute to its pathogenesis [6]. These fungus can gain entry to a susceptible host in many ways like through inhalation, ingestion of contaminated food, and through an abraded skin thus causing rhino orbital/cerebral, pulmonary, gastrointestinal, or cutaneous infections respectively. Regardless of the manifestation of the disease, a hallmark of mucormycosis is the ability of the causative organism to aggressively and rapidly invade blood vessels, which results in hematogenous dissemination, vessel thrombosis, and subsequent tissue necrosis.

Ischemic necrosis of infected tissues can prevent delivery of leukocytes and antifungal agents to the foci of infection. Therefore, interactions between invading fungi and endothelial cells lining blood vessels represent a major step in the pathogenesis of mucormycosis [1]. It is proposed that Mucorales invade the endothelium through unique receptors, the basement membranes are constituted of extracellular protein matrices that are mainly composed of laminin and collagen IV. These membranes separate epithelial or endothelial cells from underlying stroma. Due to epithelial cell damage (e.g., due to diabetes or chemotherapy), the extracellular matrix proteins can be exposed for direct interaction with inhaled or ingested spores. This attachment of the fungal spores to extracellular protein matrices is specific [1].

Clinical and experimental data demonstrate that individuals who lack phagocytes or have impaired phagocytic function are at higher risk of mucormycosis. For example, severely neutropenic patients are at increased risk for developing mucormycosis. In contrast, patients with AIDS do not seem to be at increased risk for developing mucormycosis. These findings suggest that neutrophils, 
but not necessarily $\mathrm{T}$ lymphocytes, are critical for inhibiting fungal spore proliferation. Furthermore, both mononuclear and polymorphonuclear phagocytes of normal hosts kill Mucorales by the generation of oxidative metabolites and the cationic peptides, defensins. The exact mechanisms by which phagocytes are impaired by ketoacidosis, diabetes mellitus, and corticosteroids are yet to be determined [6]. Hence Mucorales must possess unique virulence traits that enable the organism to exploit the unique state of immunosuppression and physiologic impairment seen in this subset of patients.

Iron is an essential element for cell growth and development, contributing to many vital processes of the cell. In mammalian hosts, very little serum iron is available to microorganisms because it is highly bound to carrier proteins such as transferring. Sequestration of iron by serum is a major host defense mechanism against many fungal infections. Patients with diabetic ketoacidosis have elevated levels of available serum iron, likely due to release of iron from binding proteins in the presence of acidosis. In general acidotic conditions decreased the iron-binding capacity of sera suggesting that acidosis temporarily disrupts the capacity of transferrin to bind iron therefore, the increased susceptibility to mucormycosis of patients. The increased risk of mucormycosis in patients in renal failure receiving deferoxamine iron chelation therapy is explained by the fact that deferoxamine actually acts as a siderophore for the agents of mucormycosis, supplying previously unavailable iron to the fungi.

The iron liberated from deferoxamine is likely transported into the fungus by the high affinity iron permease. In contrast, two other iron chelators, deferiprone and deferasirox, do not supply iron to the fungus and were shown to be cidal against Zygomycetes in vitro. Fungi can obtain iron from the host by using high-affinity iron permeases or low-molecular-weight iron chelators (siderophores) [7]. Thorough research of the relevant literature revealed only one study by Hosseini et al. Based on their observations in a series of 10 patients, Hosseini et al regard the pterygopalatine fossa as a reservoir of the disease through which it can spread to neighboring structures including the retroglobal space of the orbit and infratemporal fossa especially in the Rhinocerebral variant. The skin barrier represents a host defense against cutaneous mucormycosis, as evidenced by the increased risk for developing mucormycosis in persons with disruption of this barrier. The agents of mucormycosis are typically incapable of penetrating intact skin. However, burns, traumatic disruption of the skin, and persistent maceration of skin enables the organism to penetrate into deeper tissues.

Although mucormycosis can even be introduced through direct access, as has been seen with the use of contaminated tongue depressors in neonates or the use of contaminated wooden applicators used to mix drugs given to immunocompromised patients. These cases illustrate an alarming shift in mucormycosis cases from mainly community-acquired infections to nosocomial infections in susceptible hosts [6]. Other putative virulence factors include the ability of Rhizopus to secrete lytic enzymes, including aspartic proteinases. In addition, Rhizopus species have an active ketone reductase system, which may be an additional virulence factor by enhancing growth in the acidic and glucose-rich environment seen in ketoacidotic states, differences in virulence across species in the order Mucorales may also provide insight into the complex repertoire of virulence factors causing aggressive invasive disease in some species and infrequent mortality in others, despite environmental ubiquity, [6] although these are not proved as yet.

\section{Conclusion}

Mucormycosis is a serious fungal infection which although not always but predominantly involves the immunocompromised patients. As per the understanding so far these mucorales possess unique virulence traits that enable the organism to exploit the unique state of immunosuppression and physiologic impairment seen infected patients. The etiopathogenesis of the disease involves utilization of Iron hence cessation of iron in any form is important for the control of the disease. It is also known to cause endothelial damage and there is a significant difference in the prognosis of the disease with and without endothelial damage hence early antifungal therapy along with debridement of the area with endothelial damage is indicated for better prognosis. Acidotic conditions must also be treated early as they also contribute to the fungal spread, besides starting early antifungal therapy.

\section{References}

1. Clara Baldin, Ashraf S Ibrahim (2017) Molecular mechanisms of mucormycosis- The bitter and the sweet. PLoS.

2. Giulia Morace and Elisa Borghi (2012) Invasive Mold Infections: Virulence and Pathogenesis of Mucorales. International Journal of Microbiology 2012: 5.

3. K Hoffmann, J Pawłowska, G Walther, M Wrzosek, GS de Hoog, et al. (2015) The family structure of the Mucorales: a synoptic revision based on comprehensive multigene-genealogies.

4. Mucorale - Wikipedia.

5. PatrickEmanuel(2013) Mucormycosis Pathology-Dermatopathologist, Auckland, New Zealand.

6. Ashraf S Ibrahim, Brad Spellberg, Thomas J Walsh, Dimitrios P Kontoyiannis (2016) Pathogenesis of Mucormycosis.

7. Ashraf Ibrahim, Brad Spellberg, John Edwards (2016) Iron Acquisition: A Novel Prospective on Mucormycosis Pathogenesis and Treatment. 
(C) Commons Attribution 4.0 License

BY DOI: $10.19080 /$ GJO.2017.11.555812

\section{Your next submission with Juniper Publishers} will reach you the below assets

- Quality Editorial service

- Swift Peer Review

- Reprints availability

- E-prints Service

- Manuscript Podcast for convenient understanding

- Global attainment for your research

- Manuscript accessibility in different formats ( Pdf, E-pub, Full Text, Audio)

- Unceasing customer service

Track the below URL for one-step submission https://juniperpublishers.com/online-submission.php 